\title{
Internet of Plants (IoP) Empowers Bottom-up Innovations in Greenhouse Horticulture
}

\author{
Masaharu KITANO ${ }^{1}$, Koichi NomUrA ${ }^{1}$, Tomihiro YAMAZAKI ${ }^{1}$, Tadashige IwaO ${ }^{1}$, Masahiko SAItou ${ }^{1}$, \\ Makito Mori ${ }^{2}$, Daisuke Yasutake ${ }^{3}$, Takahiro KANEKO ${ }^{3}$, Hiroyuki UKedA ${ }^{4}$, Satoshi IshizuKa ${ }^{4}$, \\ Taku FUJIWARA ${ }^{2}$ and Toshihiro OKABAYASHI \\ ${ }^{1}$ IoP Promotion Office, Kochi University, Nankoku, Kochi 783-8502, Japan \\ ${ }^{2}$ Faculty of Agriculture and Marine Sciences, Kochi University, Nankoku, Kochi 783-8502, Japan \\ ${ }^{3}$ Faculty of Agriculture, Kyushu University, Fukuoka 819-0395, Japan \\ ${ }^{4}$ Center for Regional Sustainability and Innovation (CeRSI), Kochi University, Kochi 780-8073, Japan \\ ${ }^{5}$ Department of Agricultural Development, Kochi Prefectural Government, Kochi 780-8570, Japan
}

(Received February 16, 2021; Accepted June 23, 2021)

\begin{abstract}
Agriculture can be defined as a kind of the "Monodzukuri" industry (manufacturing industry) that utilizes crop photosynthesis to generate products (photosynthates). Agricultural production entirely depends on crop physioecological processes, such as photosynthesis, transpiration, translocation, and vegetative and reproductive growth, which are strongly affected by environmental conditions and farming technologies in crop fields and greenhouses. Since the beginning of agriculture, visualizing time-series data on crop physioecological processes and applying these data in everyday farm work have been impossible because of the difficulties in measuring crop physioecological processes. This invisibility of crop physioecological processes hinders not only farmers' inventive ideas but also Information and Communication Technology (ICT) and Artificial Intelligence (AI) to drive evolution and improvements in everyday farming to achieve demand-oriented crop production. Therefore, we propose an innovative concept, the "Internet of Plants (IoP)," as a regional information infrastructure for smart agriculture. This concept is driven by a cloud computing system (IoP Cloud), which is equipped with physioecological and farming support AI engines that visualize, functionalize and share highly explainable information about crop physioecological processes and farming technologies. The IoP is expected to facilitate the bottom-up evolution of agriculture, which will be driven by smart farmers empowered by IoP functions.
\end{abstract}

Keywords : Internet of Plants (IoP), cloud computing system, AI engine, physioecological processes, hybrid AI model

\section{INTRODUCTION}

Agricultural production is entirely dependent on crop physioecological processes such as photosynthesis, transpiration, translocation, vegetative growth and reproductive growth. These processes are strongly affected by various environmental conditions and farming technologies in crop production fields and greenhouses. In this context, agriculture can be defined as a kind of the "Monodzukuri" (a Japanese word for "manufacturing") industry that uses crop photosynthesis to generate products (photosynthates) with the provision of light, water, $\mathrm{CO}_{2}$, nutrients and an adequate environment for optimal crop growth. As shown in Fig. 1, the actual producers in agriculture are not the farmers but the crops, which are the primary biological producers with photosynthesizing functions; in agriculture, farmers play the role of farm managers. It is not an exaggeration to say that all work performed by farmers in crop production is performed to regulate or optimize crop physioecological processes (i.e., the production processes in the context of agriculture as a Monodzukuri industry).

In factory production, quantitative information on production processes is entirely monitored in real time with sensing systems and Information and Communications Technology (ICT). Thanks to this real-time visualization of production processes, engineers' inventive ideas and artificial intelligence (AI) can play essential roles in the innovative advancement of production processes in factories to develop optimum control and automation systems and to perform demand-oriented, just-in-time production that is optimized for quantity, quality and price. On the other hand, in crop production fields and greenhouses, the monitoring of environmental conditions, such as light intensity, air temperature, humidity and $\mathrm{CO}_{2}$ concentration, has become more common, especially in greenhouse horticulture. However, real-time information on physioecological processes, such as crop canopy photosynthesis, which is the main determinant of crop production, has remained impossible to obtain and utilize in everyday farm work since the beginning of agriculture and agricultural science. It is exceedingly difficult for not only farmers but

Corresponding author: Masaharu Kitano,

e-mail address : mkitano@kochi-u.ac.jp 


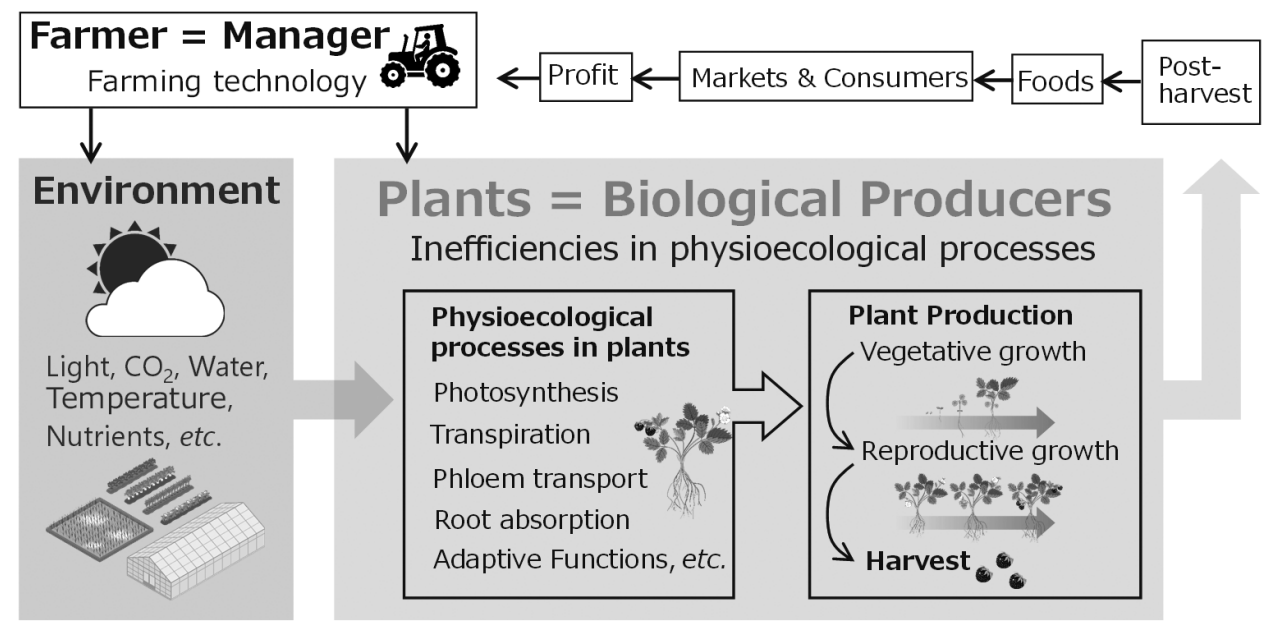

Fig. 1 Agriculture as a "Monodzukuri industry (manufacturing industry)" that is dependent on crop physioecological processes.

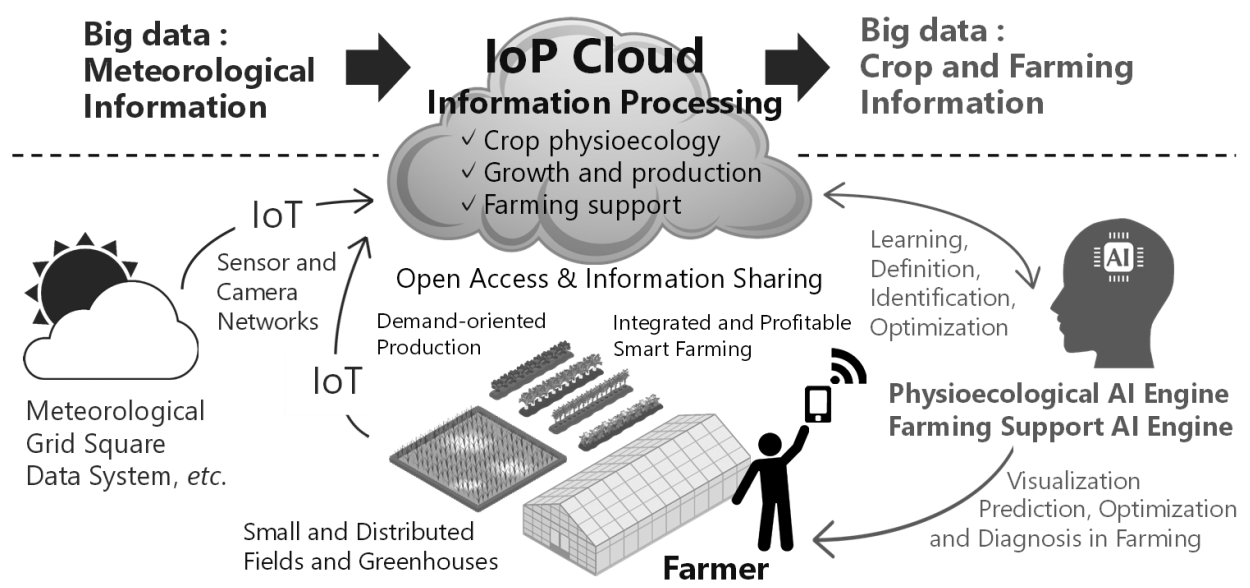

Fig. 2 An overview of the Internet of Plants (IoP) cloud computing system supported by the crop physioecological AI engine and the farming support AI engine.

also scientists to measure physioecological processes in the crop canopy at farming sites in crop production fields and greenhouses. As a result, farmers' inventive ideas, ICT and AI have not been able to drive sufficient evolutionary improvements in everyday farm work and farm management that are logically based on crop physioecological processes.

Therefore, we propose a new, innovative concept of the "Internet of Plants (IoP)" as a regional information infrastructure for smart agriculture. The IoP is expected to demonstrate that understandable and explainable information about crop physioecological processes can be visualized for everyday farm work in crop production fields and greenhouses and can then be functionalized for efficient and profitable farm management to provide demand-oriented, just-in-time production that is optimized for quantity, quality and price. Furthermore, farming technologies that are logically based on highly understandable and explainable information about crop physioecological processes can be shared within a regional group of farmers supported by the IoP and utilized for logical diagnoses of and improvements in farm work and farm management. In this paper, the innovative concept of the IoP, information processing through the IoP and the expected functions of the
IoP are described from a provocative point of view.

\section{CONCEPT OF THE IoP}

Figure 2 shows an overview of the IoP as an information infrastructure for smart farming. The IoP consists of an IoP cloud computing system (IoP Cloud) supported by a physioecological AI engine, a farming support AI engine and networks of sensors and cameras within the Internet of Things (IoT). Data on environmental conditions and images of crops in production fields and greenhouses can be easily collected by the networks of environmental sensors and crop cameras, and the meteorological grid square data system is openly accessible. These data are easily collected in IoP Cloud and are transformed into highly understandable and explainable information that is useful for everyday farm work and farm management through the smart functions (learning, definition, identification and optimization) of AI engines. IoP Cloud can visualize, functionalize and share highly understandable and explainable information; as a result, farmers can be provided with many new services for visualizing crop physioecological processes, such as canopy photosynthesis and transpiration; predicting and optimizing the crop environment, 


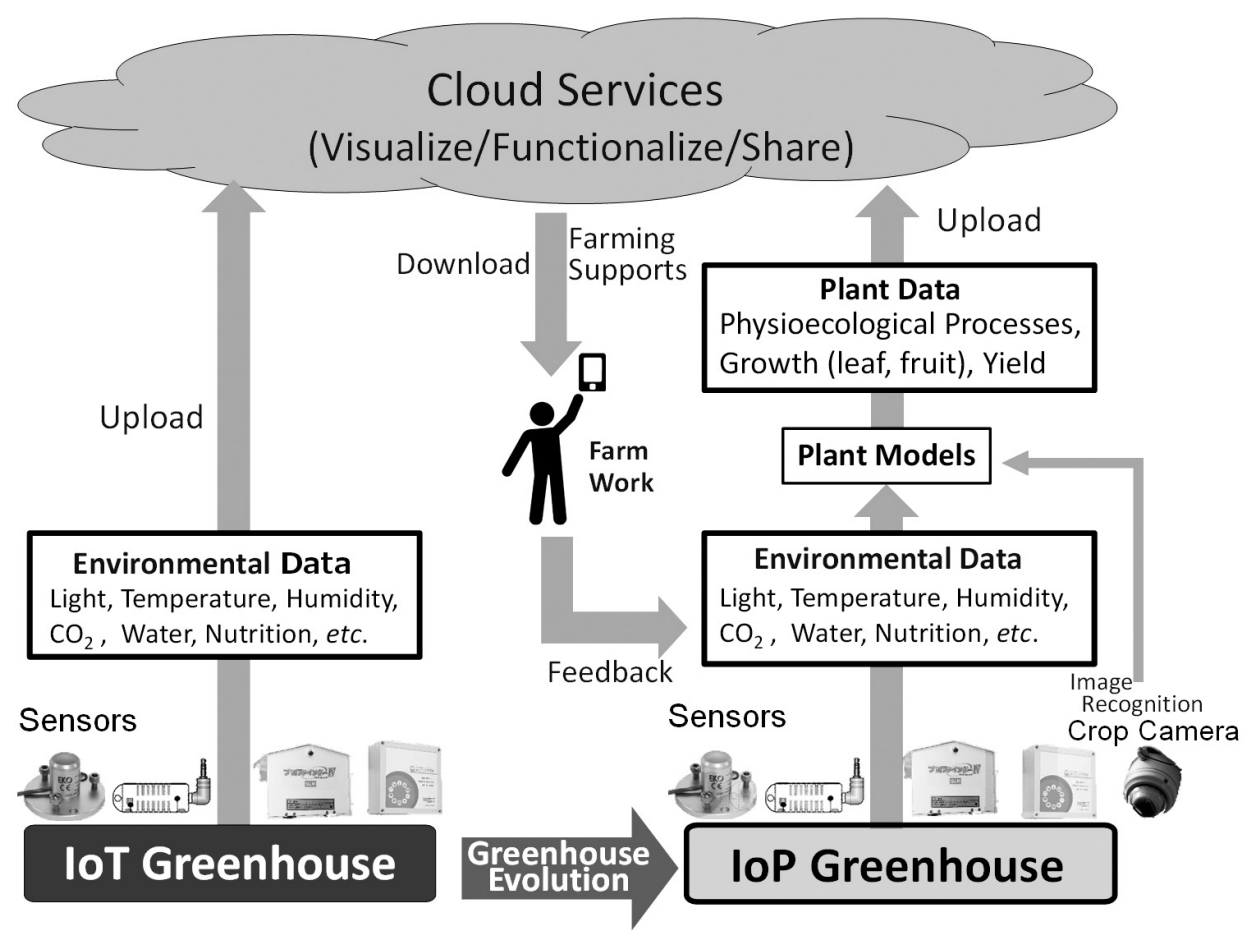

Fig. 3 Expected evolution from IoT greenhouse horticulture to IoP greenhouse horticulture.

Table 1 Understandable and explainable information about crop physioecological processes and farming support provided through the IoP visualization, functionalization and sharing functions.

\begin{tabular}{cl}
\hline Functions & \multicolumn{1}{c}{ Information on crops and farming supports } \\
\hline Visualization & $\begin{array}{l}\text { Crop physioecological processes (photosynthesis, transpiration, etc.), vegetative growth, reproductive growth } \\
\text { (flowering, fruiting, fruit growing), sugar balance, harvest (yield, date) in each greenhouse. }\end{array}$ \\
\hline Functionalization & $\begin{array}{l}\text { Prediction and optimization of environment, crop physioecological processes, vegetative growth, reproductive } \\
\text { growth (flowering, fruiting, fruit growing) and harvest (yield, date). Integrated management of distributed } \\
\text { greenhouses. }\end{array}$ \\
\hline Sharing & $\begin{array}{l}\text { Visualization, prediction and control of regional harvest. Identification and sharing of technologies of highly } \\
\text { experienced farmers. Pluralistic diagnosis and improvement of poorly experienced farmer's technology. }\end{array}$ \\
\hline
\end{tabular}

physioecological processes, vegetative and reproductive growth and yields; achieving integrated farm management of their distributed crop fields and greenhouses; and diagnosing and improving their farming technologies.

The concept of the IoP is different from that of the conventional IoT. The IoT has been defined as "a system of interrelated computing devices, mechanical and digital machines, objects, animals, or people that are provided with unique identifiers and the ability to transfer data over a network without requiring human-to-human or humanto-computer interaction (Elijah et al., 2018)." This concept was originally proposed by Ashton in 1999 (Ashton, 2009), and since then, several definitions have been proposed (Gubbi et al., 2013). The IoP, on the other hand, is defined as a series of sensors, IoP Cloud and software that can visualize, functionalize and share crop physioecological information that is useful for everyday farming and farm management. The visualization, functionalization and sharing of crop physioecological information are expected to trigger innovations in farming technology and realize the high-level standardization of farming technology among a group of farmers. While the conventional IoT mainly focuses on "data collection without human effort," the IoP emphasizes the importance of visualization, functionalization and sharing of crop physioecological information, rather than raw environmental information, which can be collected using the IoT.

In terms of future prospects for greenhouse horticulture, as shown in Fig. 3, IoT greenhouse horticulture that collects only the data from networks of sensors and cameras on the IoT should be replaced with IoP greenhouses that utilize logically explainable information about crop physioecological processes and farming support that is visualized, functionalized and shared through IoP Cloud equipped with a crop physioecological AI engine and a farming support AI engine. Table 1 shows how explainable information and farming support can be provided through the IoP functions of visualization, functionalization and sharing. The IoP can visualize physioecological processes and crop growth in each greenhouse in real time. Based on this information, the IoP can support farmers by predicting, controlling and optimizing the crop environment, photosynthesis, vegetative and reproductive growth, and yields and by allowing the integrated management of separate green- 
houses. Furthermore, the sharing of understandable and explainable information about crop physioecological processes, crop growth, farming technologies and daily farm management within a regional group of farmers is expected to drive multidimensional diagnoses and improvements and to increase the level of farming technologies used by regional groups of farmers.

\section{INFORMATION PROCESSING IN IOP}

Figure 4 shows a plan-do-check-act (PDCA) cycle of information processing between IoP Cloud and smart farmers in a greenhouse horticulture context. In each greenhouse, data on the environmental conditions (i.e., air temperature, humidity, $\mathrm{CO}_{2}$ concentration, light intensity, etc.) and the growth status of the crops are collected through networks of environmental sensors and cameras on the IoT. These easily measurable data on the greenhouse environment and the crop growth status, which reflect the farmers' technologies and performance in everyday farm work, are fed back to IoP Cloud and are transformed by the crop physioecological AI engine into logically explainable information about crop physioecological processes and vegetative and reproductive growth. Furthermore, information that provides effective farming support through visualization, prediction, control, optimization, integration and improvement is created through the farming support AI engine in combination with the physioecological AI engine. This information is then provided to smart farmers, who can exercise ingenuity in their everyday farm work with the help of the AI engines in addition to their farming knowledge and experience.

Crop physioecological AI engine

Physioecological processes and vegetative and reproductive growth in a crop plant or canopy are important aspects of everyday farm work. In particular, photosynthesis and transpiration in a crop canopy are fundamental determinants of crop production and water management, respectively. In everyday farming, however, farmers are still unable to acquire and maintain quantitative information about the rates of photosynthesis and transpiration in the crop canopy because of the difficulty of continuously measuring these parameters at farming sites. In the IoP, the crop physioecological AI engine drives the visualization of physioecological processes and growth in individual crop plants or the canopy with installed AI models.

1) Hybrid AI models installed in the AI engine

To date, tremendous efforts have been made to model crop physioecological processes (photosynthesis and transpiration in a crop plant or a canopy) and growth (flowering, fruit growth, etc.), and many models have been proposed based on the mechanistic understanding of crop physioecological processes and growth (e.g., Thornley and Johnson, 1990; Jones, 2013; Taiz et al., 2014; Ryu et al., 2019; Wallach et al., 2019). However, these mechanistic models may have limited applicability in everyday farming because they require various model parameters, some of which are not identifiable without rigorous experiments. On the other hand, AI models, which can "learn" numerical relationships between model inputs (e.g., environmental information) and outputs (i.e., crop physioecological processes and growth) in a training dataset, can directly predict outputs from inputs, without any parameter identification that requires rigorous experiments. In general, however, such AI models have a low predictive ability outside of the range of the training dataset (i.e., AI models are poor at extrapolation); thus, to be applicable to a wide range of situations, AI models require a large training dataset that covers a wide range of input and output values. To overcome these weaknesses of both mechanistic and AI models, we utilize hybrid AI models, in which mechanistic and AI models are combined (Fig. 5). In this hybrid approach, mechanistic models, based on crop physioecological principles, convert easily measurable environmental data and crop images into understandable and explainable information that has logical relationships with the output information about crop physioecological processes and growth; this converted information is fed into an AI model to generate the output information. This information processing that converts the input data into logically explainable information about crop physioecological processes and growth enables highly effective AI training, leading to high generalization of performance, even with a small amount and narrow range of training data. Such effective AI training allows us to construct and validate a powerful hybrid AI model with a small amount of input-
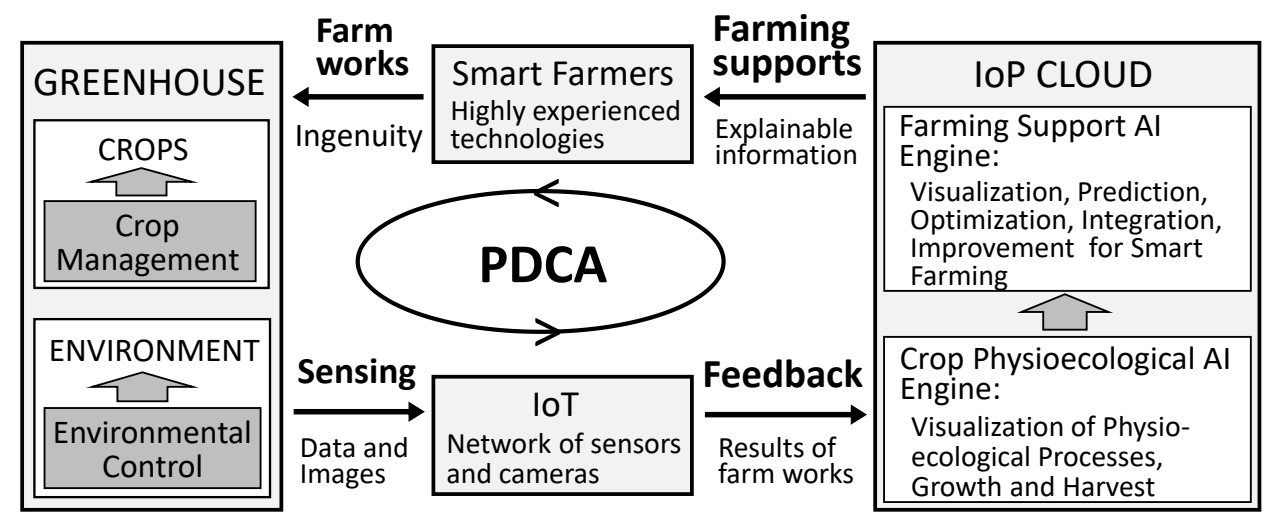

Fig. 4 PDCA cycle of information processing through IoP Cloud supported by the crop physioecological AI engine and the farming support AI engine. 


\section{Hybrid Al model in loP}

Inputs

Measurable
- Environment
Light intensity,
temperature, $\mathrm{CO}_{2}$,
humidity of air, etc.
- Crop Images

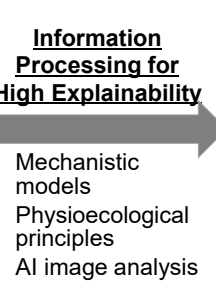

Fig. 5 Information processing in the hybrid AI model installed in the crop physioecological AI engine.

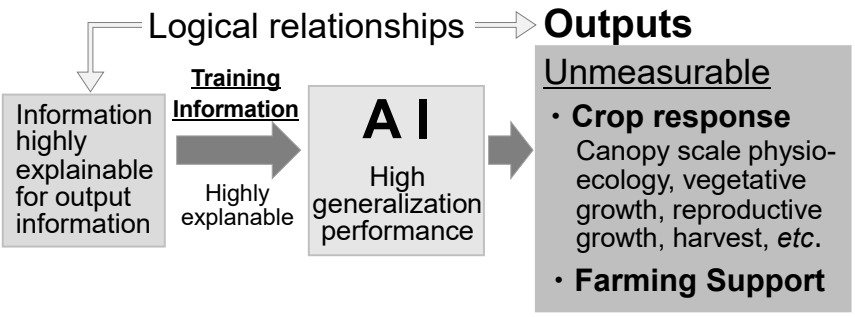

Farming Support

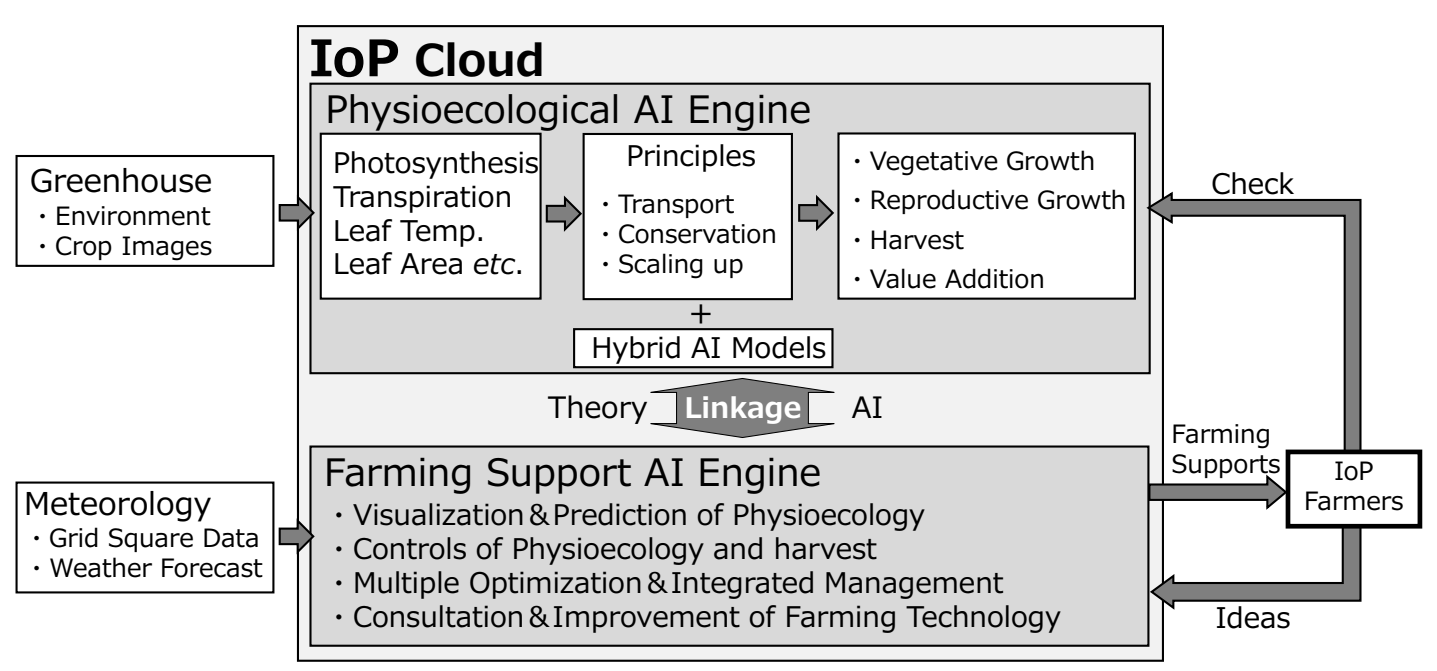

Fig. 6 Information processing in the farming support AI engine through linkage with the physioecological AI engine.

output data obtained in a much simpler experiment than the rigorous and time-consuming parameter identification. To reliably apply hybrid AI models, the two key points will be logical selection and definition of highly understandable and explainable information that comprises the output information about crop physioecological processes and growth. Such hybrid approaches have been proven to be effective in chemical engineering (Psichogios and Ungar, 1992; Thompson and Kramer, 1994; Venkatasubramanian, 2019), oil and gas production (Negash and Yaw, 2020), and several other fields (Rai and Sahu, 2020). 2) Highly understandable and explainable information in hybrid AI models

Regarding the output information on the photosynthesis and transpiration in individual crop plants or the canopy, photosynthesis and transpiration rates at the single-leaf scale can be considered the most explainable information. These rates at the single-leaf scale can be determined by solving simultaneous equations of models of crop physioecological processes, such as the biochemical photosynthesis model (Farquhar et al., 1980), the evaporative demand model (Kitano et al., 1990; Kitano and Eguchi, 1993), the models for leaf-to-air $\mathrm{CO}_{2}$ and $\mathrm{H}_{2} \mathrm{O}$ transport (Jones, 2013), the stomatal model (Medlyn et al., 2011) and the leaf temperature model (Jones, 2013). By solving these simultaneous equations with easily measurable input data on environmental conditions (light intensity, air temperature, humidity, $\mathrm{CO}_{2}$ concentration), Kimura et al. (2020) achieved the long-term visualization of the spatiotemporal distribution of the photosynthetic rate at the single-leaf scale in a multispan strawberry greenhouse. The leaf area index and irradiance at the canopy scale, which can be quantified based on canopy images extracted by applying AI image analysis, can also be considered to provide important explainable information about photosynthesis and transpiration at the canopy scale. By providing these pieces of explainable information (single-leaf photosynthetic rates and leaf area index) to an AI (artificial neural network) model predicting the canopy photosynthetic rate, it was demonstrated that the AI model's abilities for prediction and generalization were improved significantly compared with the AI model provided simply with raw environmental data (Kaneko et al., 2021; Kitano and Nomura, 2021a). Such a hybrid approach of combining crop physioecological models and an AI model was also applied successfully to estimating canopy transpiration rates (Kitano and Nomura, 2021b). With explainable information that has logical relationships with crop vegetative and reproductive growth, the hybrid AI model can be used to estimate growth rates, fruiting, yields, etc.

Farming support AI engine

Figure 6 shows the information processing in the farming support AI engine through its linkage with the crop physioecological AI engine. The farming support AI engine can visualize and predict crop physioecological processes, vegetative growth, fruiting, yields and harvest 
dates by applying hybrid AI models and mathematical models. This is carried out with the help of weather forecasts and prediction models for greenhouse environments, where highly explainable information, such as the photosynthetic rate of individual crop plants, which is provided by the physioecological AI engine, is used as one of the important explanatory variables. Furthermore, the farming support AI engine can support the control and multipurpose optimization of greenhouse environments, crop physioecological processes, growth, harvesting and farm work, as well as management, and can also provide diagnoses and improvements regarding farming technologies by applying AI technologies, such as autoencoders.

\section{FUNCTIONS OF IoP}

The IoP functions of visualization, functionalization and sharing can provide many kinds of farming support for farmers through the farming support AI engine installed on IoP Cloud. Some examples of the farming support provided by the respective IoP functions are described as follows.

\section{Visualization}

In conventional greenhouses, farmers can monitor only crop environmental conditions, such as air temperature and humidity. In IoP greenhouses, time-series of crop physioecological processes such as photosynthesis and transpiration in individual crop plants or the canopy can be visualized in real time for use in everyday farming. Figure 7 shows a sample display on a tablet visualizing timeseries (the past, the present and the near future) information about photosynthesis and transpiration rates, fruit load, flower numbers and yield in a vegetable crop. Figure 8 shows a visualization of the daily changes in photosyn- thetic rate, leaf area index, and fruit load in a fruit vegetable crop, where the fruit load is expressed as a histogram of the weight of each fruit together with its predicted harvest date. This visualized time-series information about crop physioecological processes and growth can help farm work and management achieve demand-oriented, just-intime production that is optimized for quantity, quality and price.

\section{Functionalization}

In conventional environmental control systems used in greenhouses, environmental conditions, such as the air temperature and $\mathrm{CO}_{2}$ gas concentration, are maintained within moderate ranges for the singular purpose of increasing yield. IoP Cloud with the farming support AI engine enables the optimization of environmental control for various purposes, such as controlling the rates of crop physioecological processes and growth according to a certain farming strategy, e.g., low $\mathrm{CO}_{2}$ emission. Figure 9 shows a sample display on a tablet supporting the prediction and control of the harvest date. The display can indicate the predicted daily growth and harvest date of each fruit and can also propose optimum environmental conditions, the additional cost for adjusting the harvest date, etc. Figure 10 shows a sample display on a tablet supporting the integrated monitoring and management of multiple distributed greenhouses. The farmer can check and analyze timeseries data about crop physioecological processes, growth, yield and labor in all greenhouses and establish an information-driven farm management plan and strategy. These functions of the IoP can be expected to contribute to demand-oriented, just-in-time production that is optimized for quantity, quality and price, even in the event of abnormal weather, natural disasters or pandemics.
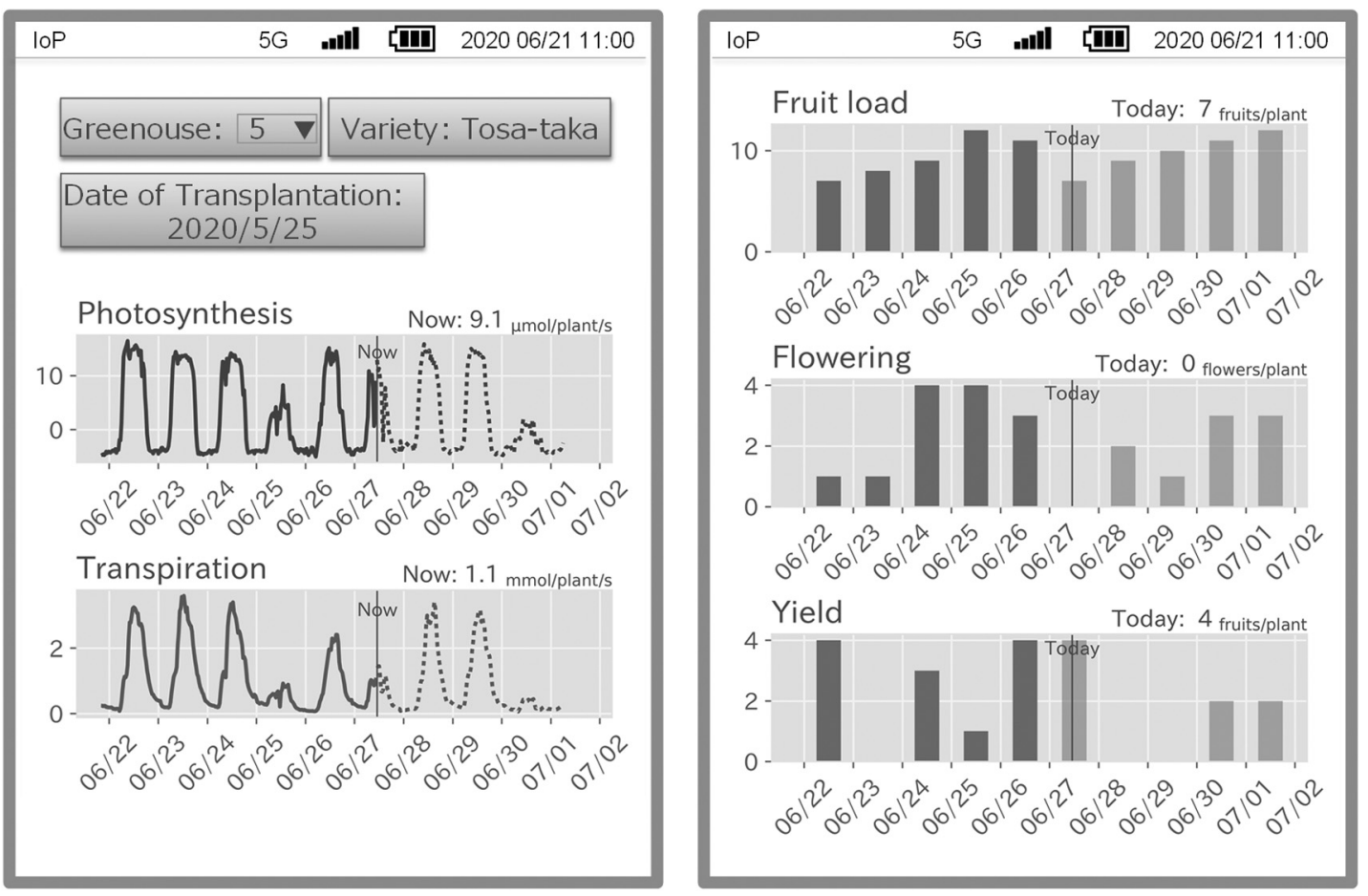

Fig. 7 Visualization of time-series data on crop physioecological processes, reproductive growth and yield. 


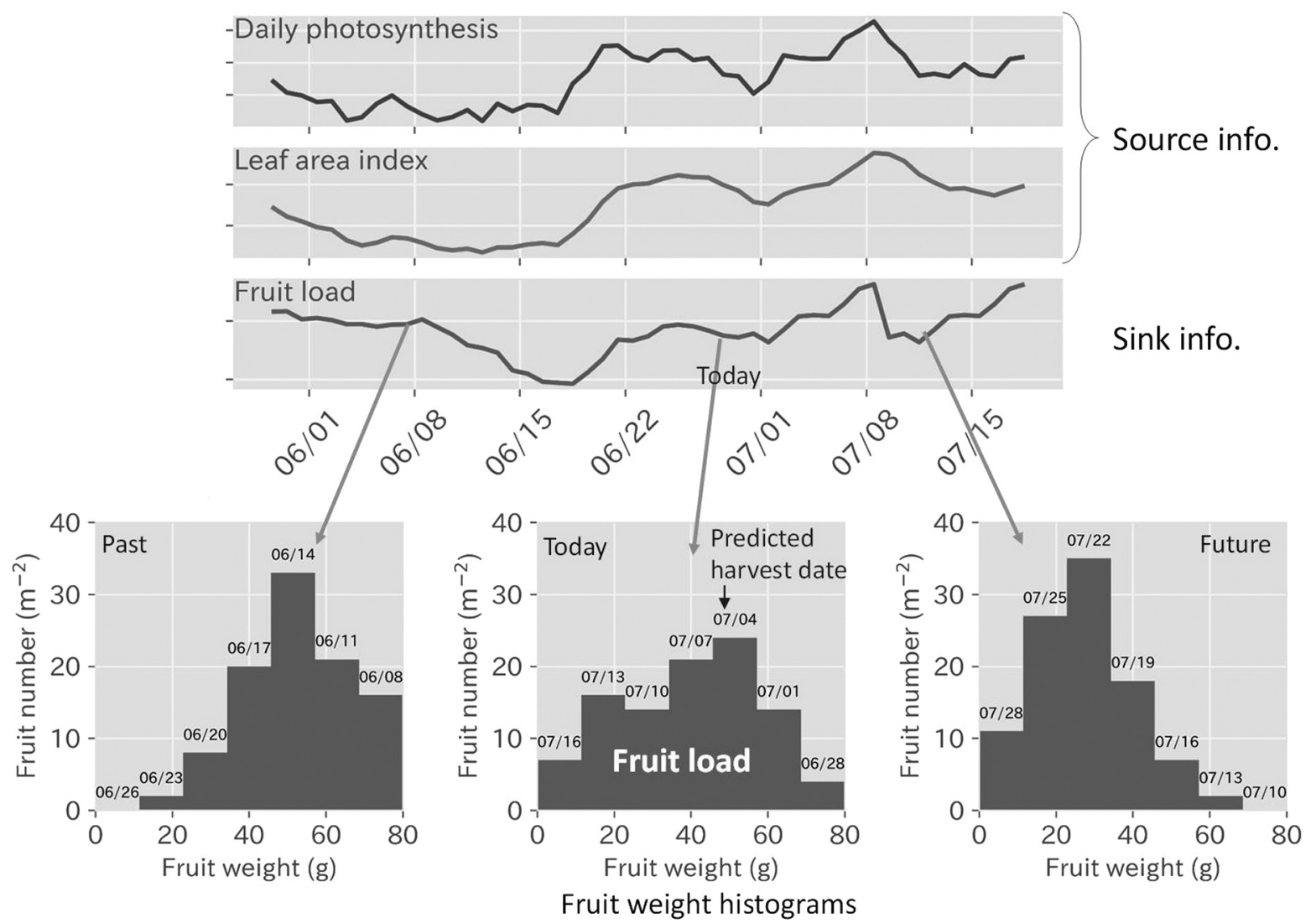

Fig. 8 Visualization of time-series data on the source-sink relationship and fruit load expressed as histograms of the weight of growing fruits.

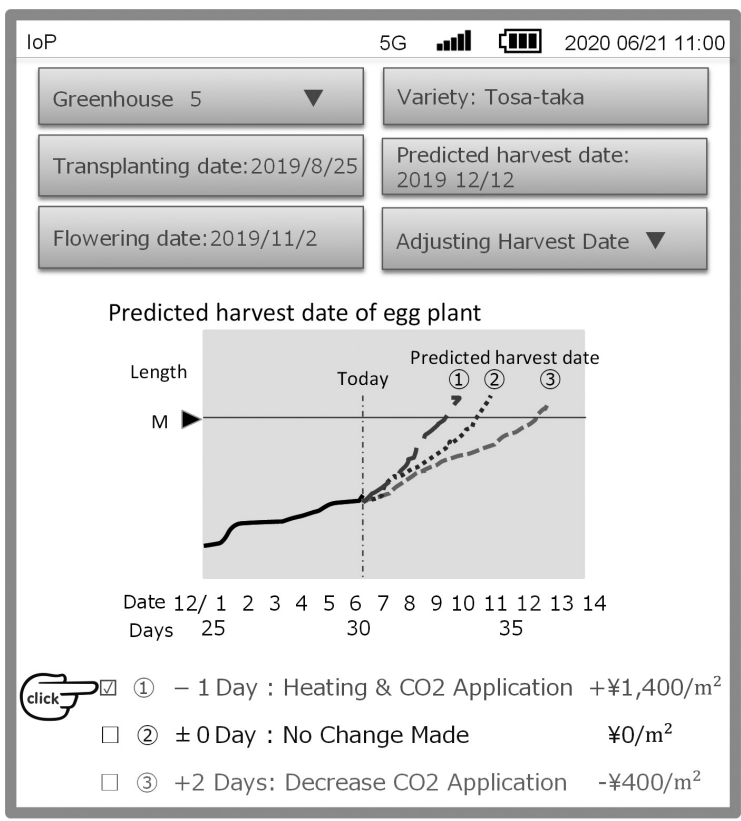

Fig. 9 Prediction and environmental optimization for harvest planning.

\section{Sharing}

IoP Cloud allows a group of farmers to share information (environmental conditions and crop physioecological processes and growth) and farming technologies in their respective greenhouses and to improve their farming technologies by comparative analyses, making full use of ICT and AI. Figure 11 shows a schematic description of the evolution of high-level farming technology standardization among a group of farmers. By identifying excellent farming technologies and sharing these technologies within the farmer group, the level of farming technology in the farmer group can evolve autonomously, from lower levels with great variation to higher levels with little variation. Figure 12 shows a schematic diagram of the consultation support for the improvement of farmer technology provided by the AI autoencoder. The higher-dimension input data, such as time-series of crop images, crop environmental conditions, crop physioecological processes (photosynthetic rate, transpiration rate, leaf temperature, vegetative growth, reproductive growth, yield, etc.) in each farmer's greenhouse, which reflect the farming technology used by each farmer, can be compressed into a lower-dimensional feature vector encoded by the AI autoencoder. Comparing the compressed feature vectors from the greenhouses of each farmer with the feature vector from the best farmer and decoding the differences would allow individual farmers to investigate the inferior aspects of their farming technology from various perspectives and to specifically improve their farming technology.

\section{GOALS OF IoP}

Farmers, governments (administrative agencies), academia (universities and research institutions) and industries relevant to agriculture should be responsible for the development and improvement of the crop supply chain from 

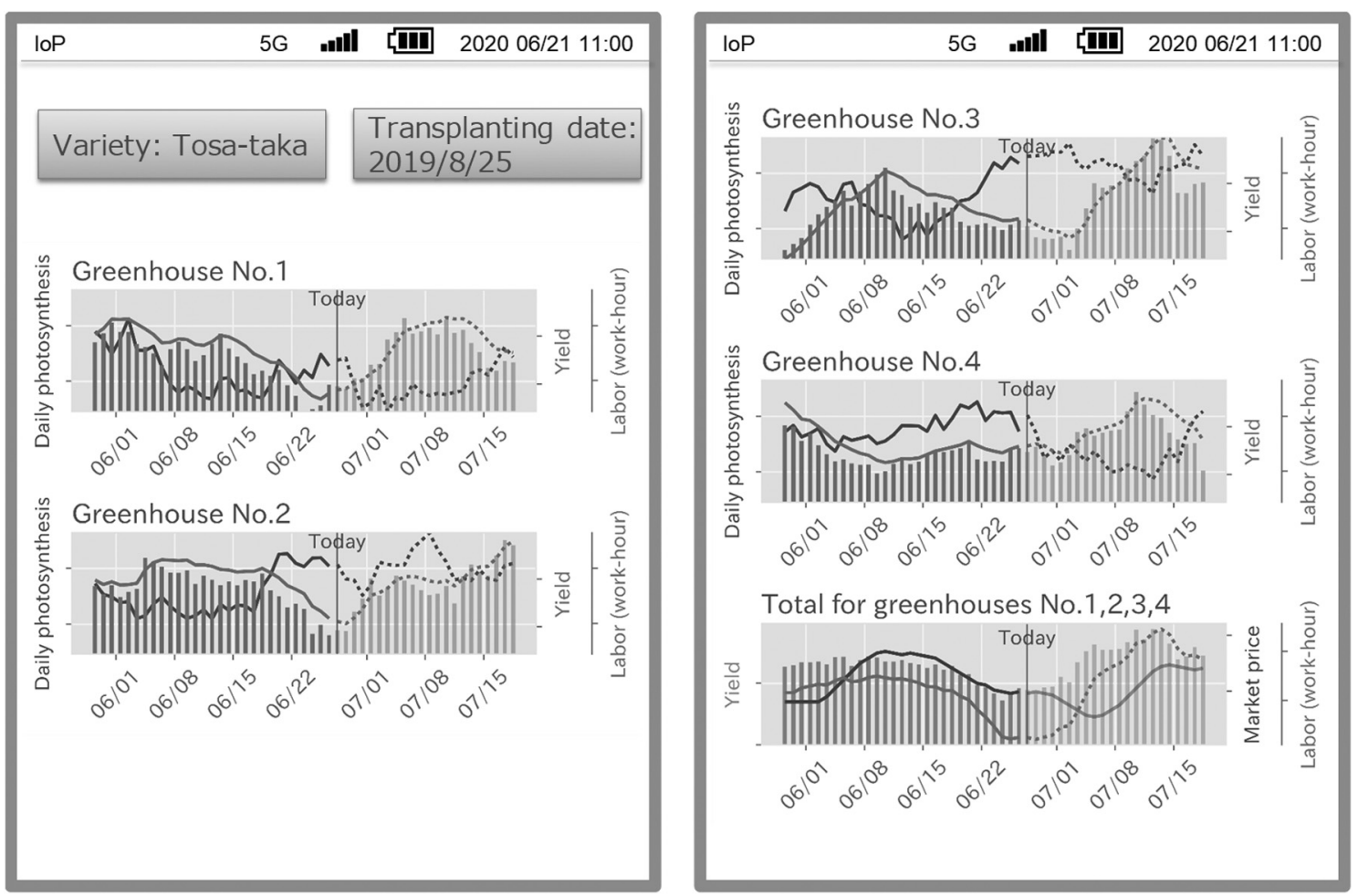

Fig. 10 Integrated monitoring and management of multiple distributed greenhouses.

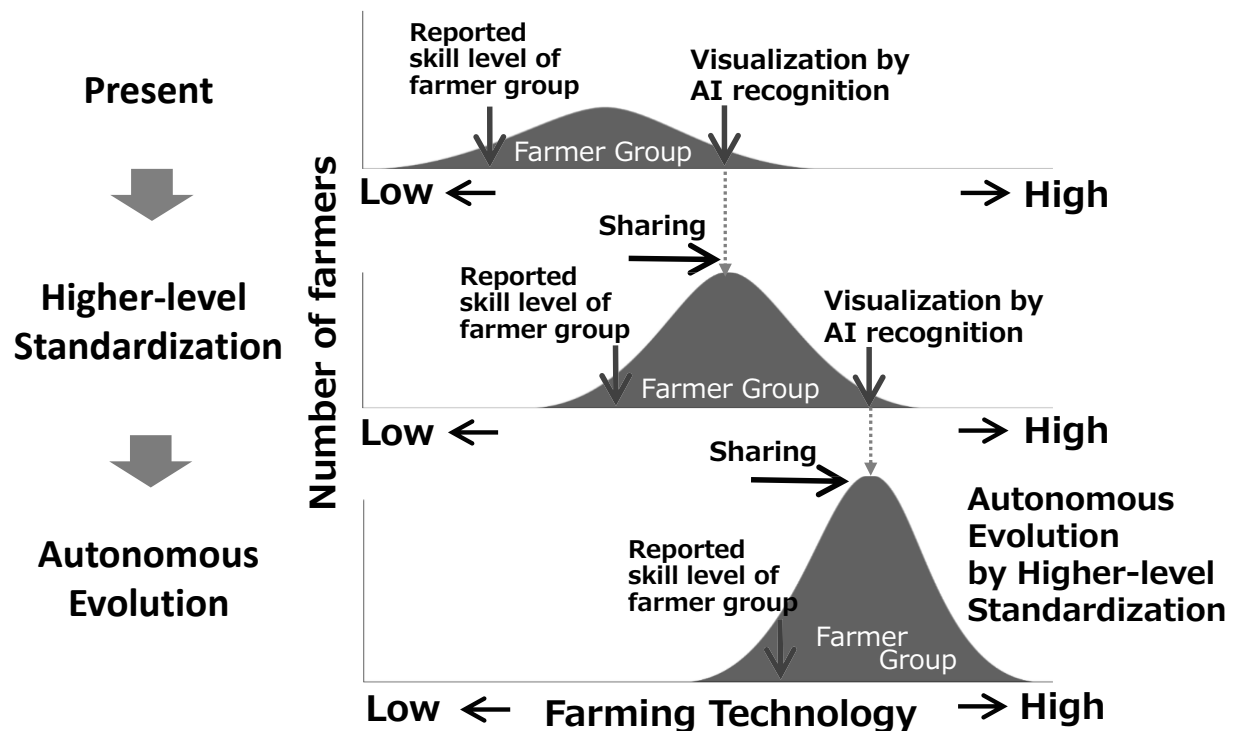

Fig. 11 Autonomous evolution toward high-level standardization of farming technology in a group of farmers through the identification and sharing of excellent farmer's technology.

production to consumption. However, agriculture has been governed, supported and improved mainly on a top-down basis by policies and research from governments and academia, despite the fact that officials and scientists from these institutions are not directly engaged in the practical farm work involved in crop production. In the recent history of agriculture, crop-producing farmers have hardly been involved in agricultural evolution and innovation from the bottom up. This situation seems to have caused a discrepancy between agricultural research in governments and academia and what farmers expect/want from research.
IoP Cloud, as an agricultural information infrastructure, is expected to drive bottom-up evolution and innovations from smart farmers by visualizing, functionalizing and sharing understandable, explainable, and useful online information about their crops and everyday farm work. Such bottom-up, farmer-driven evolution of and innovation in agricultural technology are different from the topdown, conventional development of farming technology based on policies and research from governments and academia. Figure 13 shows a symbolic mechanism representing the autonomous evolution of agriculture and the 


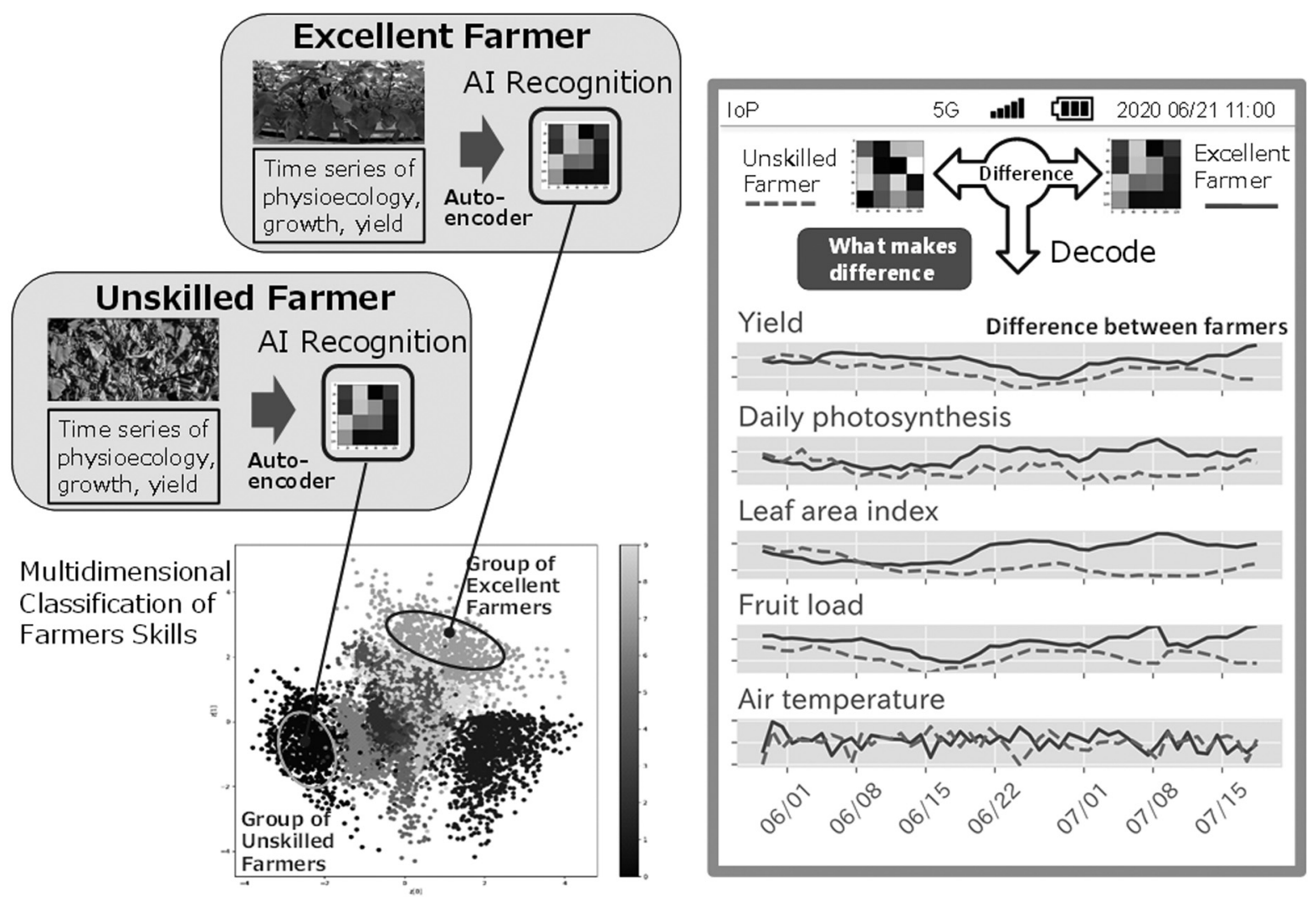

Fig. 12 Consultation support to improve farmer technologies by applying AI autoencoder technology.

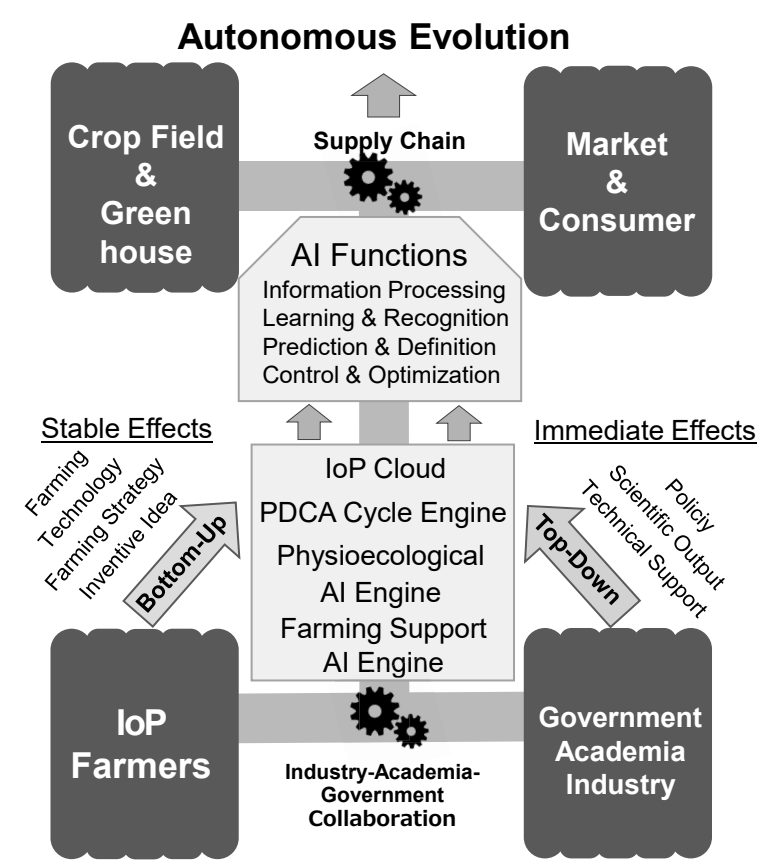

Fig. 13 Schematic vision of the autonomous evolution of agriculture and the food supply chain driven by the empowerment of IoP farmers to develop bottom-up innovations.

food supply chain driven by the IoP, which is equipped with AI engines. The evolution mechanism is driven by the physioecological and farming support AI engines on IoP Cloud and is empowered by IoP farmers on a bottom-up basis, as well as by governments, academia and industries on a top-down basis. In conjunction with AI functions, such as information collection, learning, recognition and optimization, IoP Cloud can evolve autonomously through the PDCA cycle, with bottom-up empowerment from farmers and top-down support from governments, academia and industries.

Through bottom-up empowerment from IoP smart farmers, IoP Cloud can evolve over time into a regional and industrial information infrastructure. Moreover, the system can be expected to provide the regional agriculture and food supply chain with high adaptability and resilience against the intensified damage brought by abnormal weather, severe natural disasters and pandemics, which are anticipated to occur more frequently in the future.

IoP Cloud is now under construction for highly intensive greenhouse horticulture in Kochi prefecture, Japan, which has been the national champion of greenhouse horticulture achieving the highest land productivity and also a challenger progressively facing up to serious problems in agriculture for future generations.

\section{ACKNOWLEDGMENTS}

This study was supported by a Cabinet Office grantin-aid, the Advanced Next-Generation Greenhouse Horticulture by IoP (Internet of Plants), Japan. 


\section{KITANO ET AL.}

\section{REFERENCES}

Ashton, K. 2009. That 'Internet of Things' thing. RFID J. 22: 97-114.

Elijah, O., Rahman, T. A., Orikumhi, I., Leow, C. Y., Hindia, M. N. 2018. An overview of Internet of Things (IoT) and data analytics in agriculture: benefits and challenges. IEEE Internet Things J. 5: 3758-3773.

Farquhar, G. D., von Caemmerer, S., Berry, J. A. 1980. A biochemical model of photosynthetic $\mathrm{CO}_{2}$ assimilation in leaves of C3 species. Planta 149: 78-90.

Gubbi, J., Buyya, R., Marusic, S., Palaniswami, M. 2013. Internet of Things (IoT): a vision, architectural elements, and future directions. Futur. Gener. Comput. Syst. 29: 1645-1660.

Jones, H. G. 2013. Plants and Microclimate. Cambridge University Press, Cambridge.

Kaneko, T., Nomura, K., Yasutake, D., Hirota, T., Iwao, T., Okayasu, T., Ozaki, Y., Kitano, M. 2021. Hybrid AI model for estimating canopy photosynthesis II. Verification of validity and generalizability under various environmental conditions. International Symposium on Agricultural Meteorology, March, Sapporo, Int. Symp. Agric. Meteorol. (abstract) pp 125.

Kimura, K., Yasutake, D., Koikawa, K., Kitano, M. 2020. Spatiotemporal variability of leaf photosynthesis and its linkage with microclimates across an environment-controlled greenhouse. Biosyst. Eng. 195: 97-115.

Kitano, M., Eguchi, H. 1993. An impact coefficient of evaporative demand on plant water balance. Biotronics 22: 61-72.

Kitano, M., Hamakoga, M., Eguchi, H. 1990. Physical evaluation of effective evaporative demand with reference to plant water relations. Biotronics 19: 109-119.

Kitano, M., Nomura, K. 2021a. Japan Patent Application
JP2021-019532. Japan.

Kitano, M., Nomura, K. 2021b. Japan Patent Application JP2021-062642. Japan.

Medlyn, B. E., Duursma, R. A., Eamus, D., Ellsworth, D. S., Prentice, I. C., Barton, C. V. M., Crous, K. Y., De Angelis, P., Freeman, M., Wingate, L. 2011. Reconciling the optimal and empirical approaches to modelling stomatal conductance. Glob. Chang. Biol. 17: 2134-2144.

Negash, B. M., Yaw, A. D. 2020. Artificial neural network based production forecasting for a hydrocarbon reservoir under water injection. Pet. Explor. Dev. 47: 383-392.

Psichogios, D. C., Ungar, L. H. 1992. A hybrid neural networkfirst principles approach to process modeling. AIChE J. 38: 1499-1511.

Rai, R., Sahu, C. K. 2020. Driven by data or derived through physics? A review of hybrid physics guided machine learning techniques with cyber-physical system (CPS) focus. IEEE Access 8: 71050-71073.

Ryu, Y., Berry, J. A., Baldocchi, D. D. 2019. What is global photosynthesis? History, uncertainties and opportunities. Remote Sens. Environ. 223: 95-114.

Taiz, L., Zeiger, E., Møller, I. M., Murphy, A. 2014. Plant Physiology \& Development. Sinauer Associates, Massachusetts.

Thompson, M. L., Kramer, M. A. 1994. Modeling chemical processes using prior knowledge and neural networks. AIChE J. 40: $1328-1340$.

Thornley, J. H., Johnson, I. R. 1990. Plant and Crop Modelling. Clarendon Press, Oxford.

Venkatasubramanian, V. 2019. The promise of artificial intelligence in chemical engineering: is it here, finally? AIChE J. 65: 466-478.

Wallach, D., Makowski, D., Jones, J. M., Brun, F. 2019. Working with Dynamic Crop Models. Elsevier, Amsterdam. 